\title{
Study on the Scale Determination of Urban Rail Transfer Station
}

\author{
Yuping Wang ${ }^{1,3, a}$, Yaping Zhang ${ }^{3, b}$, Huizhi $X u^{2,3, c}$ \\ ${ }^{1}$ The Preparation Research Centre, Harbin Urban and Rural Planning Bureau, Harbin, 150000, \\ China $^{2}$ Harbin Metro Group Corporation Ltd, Harbin, 150000, China, ${ }^{3}$ Harbin Institute of
}

Technology, Harbin, 150090, China

a wangyuping004997@163.com, ${ }^{\mathrm{b}}$ 895679648@qq.com, ${ }^{\mathrm{c}}$ 353812396@qq.com

Key words: urban rail transit, transfer station, facilities, scale

\begin{abstract}
As the major distributing center and intermediate transit point, the scale of transfer station in urban rail transit system directly affects the operational efficiency and overall cost of the entire system. So, accurately controlling the scale of transfer station becomes one of the most important aspects in improving service level and reducing the overall project cost. On the basis of summarizing the method on determining the scale of transfer station both home and abroad, the paper describes the role of the various facilities in rail transfer station, and illustrates the problems of our rail transfer station. Following the above discussion and investigation, the sizes of typical transfer station facilities are discussed and improved (e.g. vertical elevator). Taking the Longjiang Street station as an example, the proposed methods and models are verified and the analysis result shows that this transfer station should use parallel transfer mode.
\end{abstract}

\section{Introduction}

With the improvement of urbanization level, the serviceability of public transport facilities is becoming harder and harder to adapt to the increasing travel demands. Thus, more and more cities in China begin to turn their attention to urban rail transit system, which has the characters of high speed, large volume and high punctuality rate. However, because of the particularity, the construction cost of the urban rail transit is huge. As the important node for passengers to gather and leave, the scale of transfer station will directly impact the operational efficiency and overall cost of the entire system. So, accurately controlling the scale of transfer station becomes one of the most important aspects in improving service level and reducing the overall project cost.

Vladimir ${ }^{[1]}$ established 0-1 Linear Programming model, which was suitable for the determination of airport scope. Bates ${ }^{[2]}$ introduced the function and form of the transfer facilities, elaborated the right scale of each kind of transfer station, and discussed the improvement measures of transfer facilities. Dickins ${ }^{[3]}$ gave some recommendations on the location, layout and scale of inner facilities, and summarized the factors that affected efficient use of the facilities by investigating the transit rail systems of 51 cities in Europe and South America. Miclea ${ }^{[4]}$ studied the scale of the pedestrian facilities underground from the security point of view, and proposed the minimum design standards meeting the safety need of every facility. Shen Jingyan ${ }^{[5]}$ analyzed dynamic variation of passengers on the platform, concluded the distribution graphics and related parameters, studied the reasonable scale of platform, gave simple calculation method. Tan $\mathrm{Yu}^{[6]}$ studied the demands of facilities, the transfer form, and the connection between rail transit and other transportation from the single station point of view. Wang Xiaorong ${ }^{[7]}$ proposed implementation doctrine of public zone, platform and equipment rooms. 
Following the above discussion, the sizes of typical transfer station facilities are discussed and improved (e.g. vertical elevator, automatic fare collector). Taking the Longjiang Street station (connecting Harbin Metro Line $1 \&$ Line 5 together) as an example, the proposed methods and models are verified and the analysis results show that this transfer station should use parallel transfer mode.

\section{Inner facilities in transfer station}

In principle, transit rail station consists of waiting room, platforms, station buildings, passageways and stairs. The platform is the most basic part which must be set. Other parts usually should be set, and sometimes they might be simplified for some special condition or meeting the function.

Waiting room. The function of waiting room is guiding the passengers along the way from platform to gateway quickly, safely and conveniently. It is transitional space for passengers boarding. So, it is necessary to set Automatic Fare Collection System and query facilities. Besides, there are other facilities in the waiting room need to be set, such as equipment and management room, and lifting equipment.

Platform. The platform is the site providing boarding and waiting space for passengers, and it is one of the most important parts in the rail transit system facilities. The platform could be set either above, under or parallel to the waiting room, and can be classified into two kinds, island platform and side platform, according to the layout form of the station.

Automatic Fare Collection System. AFC can realize the processes of booking, checking, and charging automatically, which would not only reduce the time of passengers' ticket into station, but also improve the efficiency of the station management to some extent. The scale of AFC should meet the need of serving passengers. It is should also be avoided too much configuration, which would result in a waste of construction costs.

Vertical mobile facilities. Vertical movement facilities include stairs, escalator, elevator and incline, which are mainly set up at the gateway and fee area. The incline is usually set up to serve the handicapped and people with big luggage ${ }^{[8]}$.

\section{Determination models for transfer station scale}

Determination of platform scale. The size of the platform refers to the width, length and height. The length should at least meet the need of train stopping. It can be calculated by formula (1).

$$
L=L_{0}+L_{u}
$$

$L$ is the length of platform, $L_{0}$ is the length of marshalling in the future, and $L_{u}$ is uncertain distance of the train stopping allowed, usually taking 4 to 10 meters.

The width of platform is determined by the passenger volume during peak hours in the future, platform type, layout of station house, stairs, escalator, and so on, meeting the least width.

Platform is divided into island and side. If the width of side platform is $B_{c}$, the width of island platform will be as follows.

$$
W_{d}=2 B_{c}+n \cdot W_{z}+W_{l}
$$

$W_{z}$ means the width of an upright, $W_{l}$ means the width of the stair or the escalator, and $n$ is the number of uprights in the direction of platform cross section. Before the train arriving, the main function of the platform is waiting. Assuming passengers are uniformly distributed, not occupying the width of safety area ${ }^{[5]}$. Then the width of platform can be calculated as follows: 


$$
W_{c 1}=\left(Q_{i n}+Q_{\text {transfer }}\right) \beta /\left(q \cdot L \rho_{1}\right)+W_{a}
$$

$W_{c l}$ means the calculated width of platform before the train arriving, $Q_{i n}$ means the passenger volume pulling in during peak hours, and $Q_{\text {transfer }}$ means the passenger volume need to transfer. $\beta$ is the peak hour factor, usually taking 1.2 to 1.4. $q$ is the rate of train flow, relevant to departure interval time. $L$ means the effective length of the platform, $\rho_{1}$ is the standing density of passengers before the train arriving, and $W_{a}$ is the width of safety area. When the train arrives, passengers getting on will crowd at both sides of the door. Then the length of passengers on the platform equals to the effective length of platform subtract sum of all doors' width. The width of waiting area on the platform when the train arriving is as follows:

$$
W_{c 2}=\left(Q_{i n}+Q_{\text {transfer }}\right) \beta / q \cdot(L-n b) \rho_{2}+W_{a}
$$

$n$ means the number of marshalling, $b$ is the width of each door, and $\rho_{2}$ means the standing density of passengers when the train arriving. The difference between the widths before the train arriving and when arriving, can be seen as the width of passageway for passengers getting off the train.

$$
W_{c 3}=W_{c 1}-W_{c 2}
$$

Usually the width of side platform should meet the following conditions. That is:

$$
W_{c 3}=W_{c 1}-W_{c 2} \geq 1.0, \quad W_{c 1} \geq W_{c 2}+1.0
$$

The transfer style can be classified into three kinds: side with side, island with island and island with side, just as figure 1 to figure 3 show. Assuming that there are two lines interweaved together, Line $\mathrm{M}$ and Line $\mathrm{L}$. The width of platform transferring on the same layer can be divided into six parts: the width of waiting area for Line M uplink, the width of waiting area for Line L uplink, the width of waiting area for Line M downlink, the width of waiting area for Line L downlink, the width of stairs or escalator and the width of uprights. The passenger volumes are different for different waiting areas. The width of platform for island with island transfer style is as follow:

$$
W_{1}=\left(Q_{M U}+Q_{M D}+Q_{L U}+Q_{L D}\right) \beta /\left(q \cdot L \rho_{1}\right)+4 W_{a}+n W_{z}+2 W_{l}
$$

In a similar way, the width of platform for island with side transfer style is as follow:

$$
W_{2}=\left(Q_{M U}+Q_{M D}+Q_{L U}+Q_{L D}\right) \beta / q \cdot\left(L \rho_{1}\right)+4 W_{a}+n W_{z}+3 W_{l}
$$

$Q_{M U}$ refers to passenger volume for Line M uplink, $Q_{M D}$ refers to passenger volume for Line M downlink, $Q_{L U}$ refers to passenger volume for Line L uplink, and $Q_{L D}$ refers to passenger volume for Line 1 downlink.

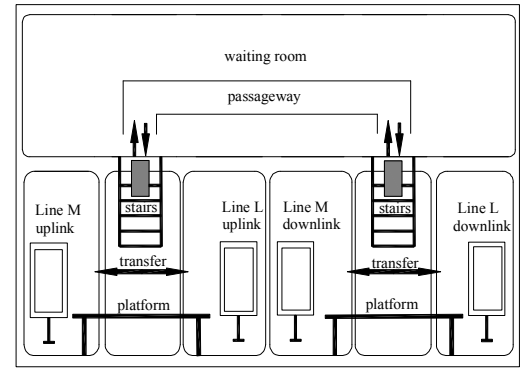

(a) island with island $W_{1}$

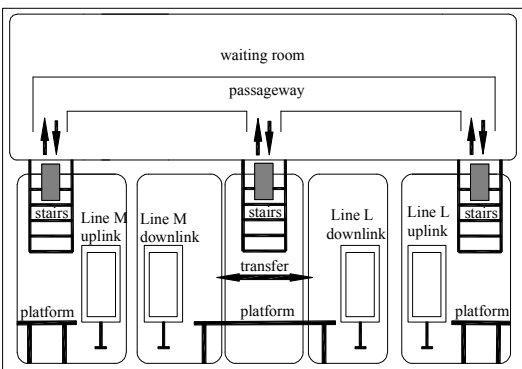

(b) island with side $W_{2}$

Figure 1 Parallel transfer on the same platform 
There is another transfer style on the different layers, just as figure 2 shows. The widths of platform transferring on different layers can be calculated as follows:

$$
\begin{aligned}
& W_{3}=\left(Q_{M U}+Q_{L U}\right) \beta /\left(q \cdot L \rho_{1}\right)+2 W_{a}+n W_{z}+W_{l} \\
& W_{4}=\left(Q_{M D}+Q_{L D}\right) \beta /\left(q \cdot L \rho_{1}\right)+2 W_{a}+n W_{z}+W_{l}
\end{aligned}
$$

$W_{3}$ refers to the platform width on the second floor, and $W_{4}$ refers to the platform width on the third floor. The final width of the platform should take the maximum of the two widths.

$$
W_{5}=\max \left\{W_{3}, W_{4}\right\}
$$

Besides, there is another kind of transfer style, node transfer. There are three layers in such station, and different rail lines are located at different layers, linked with each other by stairs or escalator, just as figure 3 shows.
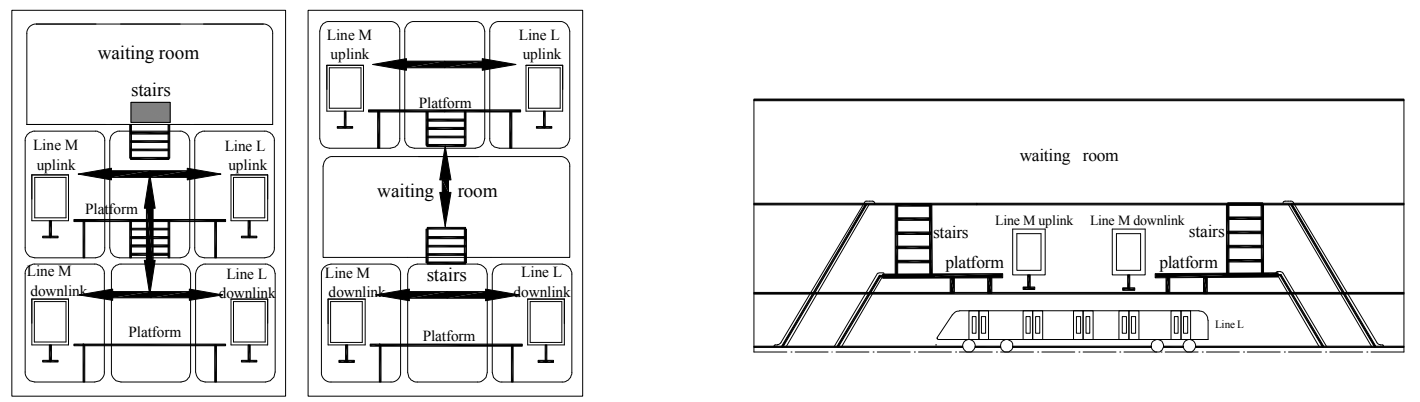

Figure 2 Parallel transfer on the different floor layers

Figure 3 Platform for node transfer style

Width of platform on the second floor underground:

$$
W_{6}=\left(Q_{M U}+Q_{M D}\right) \beta /\left(q \cdot L \rho_{1}\right)+2 W_{a}+2 W_{l}
$$

Width of platform on the third floor underground:

$$
W_{7}=\left(Q_{L U}+Q_{L D}\right) \beta /\left(q \cdot L \rho_{1}\right)+2 W_{a}+n W_{z}+W_{l}
$$

Determination of vertical mobile facilities scale. The number and width of stairs or escalators should meet the requirements for timely evacuation. They are also connected with delivery capacity and passenger volumes during peak hours in the future ${ }^{[9]}$.

The width of stairs should be determined by the passenger volumes both getting on and off the trains, on the basis of meeting disaster prevention requirements.

The width of stairs shown in figure 1 can be calculated as follows:

$$
W_{l}=\left(Q_{l U}+Q_{I D}\right) \beta / N C+W_{f}
$$

$W_{l}$ is the width of stairs, $Q_{l U}$ and $Q_{l D}$ are passenger volumes for uplink and downlink respectively. $C$ is the capacity of per meter of stair for passenger both up and down. $\beta$ refers to peak hour factor, usually taking between 1.2 and $1.4, N$ is the number of stairs, and $W_{f}$ is the width of stair railing.

The number of escalators can be calculated by the following formulas.

$$
N_{U}=Q_{U} \beta / C k, \quad N_{D}=Q_{D} \beta / C k
$$

$N_{U}, N_{D}$ are the number of escalators for uplink and downlink respectively. $Q_{I U}$ and $Q_{I D}$ are passenger volumes for uplink and downlink. $k$ is the utilization of the escalator per hour, usually taking 0.8 . 
Determination of AFC scale. The main equipments of AFC are the fare gates for getting in and out of the station. They are determined according to the passenger volumes during peak hours, just as follows.

$$
N_{I N}=I N T\left[Q_{\text {in }} \beta / C_{I}\right], \quad N_{\text {OUT }}=I N T\left[Q_{o u t} \beta / C_{O}\right]
$$

$N_{I N}, N_{\text {OUT }}$ are the number of fare gates for in and out respectively. $Q_{I N}, Q_{O U T}$ are the passenger volumes getting in and out of the station. $C_{I}, C_{O}$ are ticket checking capacities.

\section{Case}

Taking Longjiang Street Station in Harbin for example. The designers designed two kinds of transfer styles for the station, parallel transfer on the same side of platform and cross-shaped node transfer on different layers, just as figure 4 shows.

The passenger volumes of Line one and Line five are 33500 persons per hour and 20900 persons per hour respectively. Assuming that it takes B type vehicle, with four gates on each side, and 1.2 meters for each gate. And the width of each escalator is one meter, and the utilization is 0.8 . The utilization of ticket vendors in the station is sixty percent

Platforms of different transfer styles have different length, width and escalator number, just as Tab. 1 and Tab. 2 show. From the result, it can be concluded that the scale of platform transferring on the same side is less than the scale of platform transferring through node on different layers. And besides, there are only two layers of floors in the platform transferring on the same side. So the paper recommends the former as the station style in Longjiang Street Station.

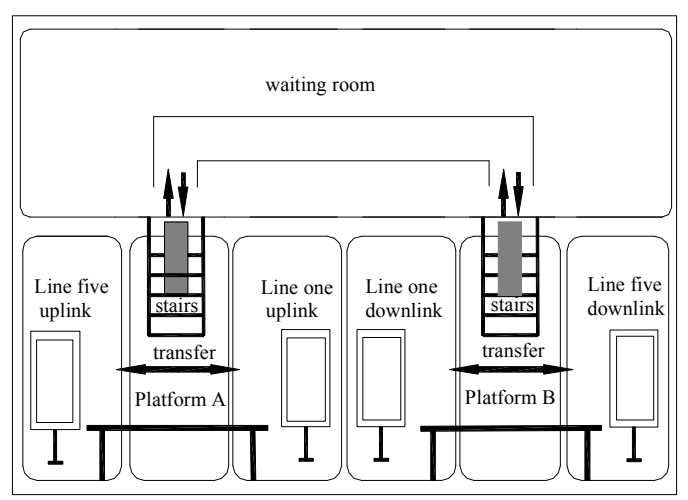

(a) parallel transfer

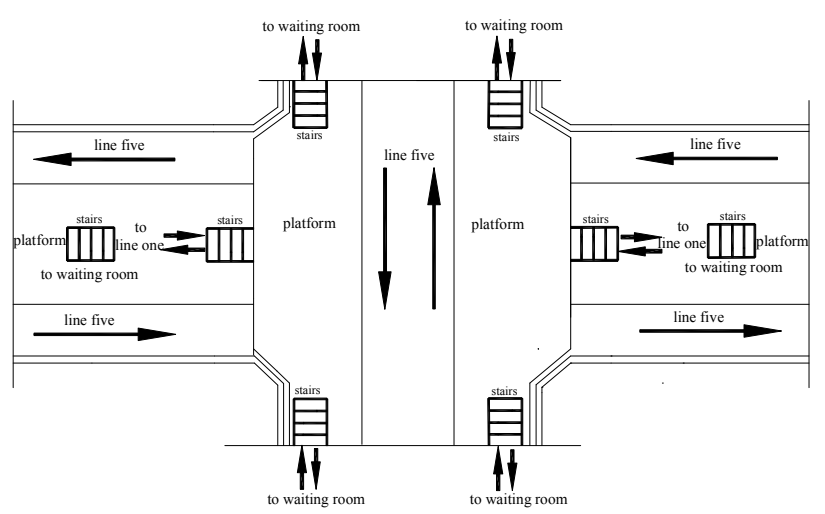

(b) cross-shaped node transfer

Figure 4 The station style in Longjiang Street Station

\section{Conclusion}

The paper proposes scale determination methods of typical facilities in transfer station for both parallel transfer and node transfer. The study improves the calculation methods of the sizes for platform, stairs, escalators ticket vendors, and checkers. Taking the Longjiang Street station as an example, the case study proves the validity and operability of the proposed method. 
Tab. 1 The scale of facilities for different transfer styles (platform and stairs)

\begin{tabular}{c|c|c|c|c}
\hline \hline \multirow{2}{*}{ Transfer style } & \multicolumn{2}{|c|}{ platform } & \multicolumn{2}{|c}{ stairs } \\
\cline { 2 - 5 } & length & $\begin{array}{c}\text { Width(with } \\
\text { stairs and escalators) }\end{array}$ & number & width \\
\hline parallel transfer & $96 \sim 102$ & 21.15 & 4 & 11.1 \\
\hline \multirow{2}{*}{ node transfer } & $96 \sim 102$ & 21.74 & 8 & 18.3 \\
\cline { 2 - 5 } & $82 \sim 88$ & & 8 \\
\hline \hline
\end{tabular}

Tab. 2 The scale of facilities for different transfer styles (other facilities)

\begin{tabular}{c|c|c|c}
\hline \hline Transfer style & escalator qualities & ticket vendors & ticket checkers \\
\hline parallel transfer & 5 & 20 & 17 \\
\hline node transfer & 6 & 20 & \\
\hline \hline
\end{tabular}

\section{Acknowledgements}

The authors would like to thank Harbin Metro Group Corporation Ltd. and Harbin Urban and Rural Planning Bureau for providing the useful data about the rail transit.

\section{References}

[1] VlmadimiMrarinao, DnaielSerra, Location of Hubsina Competetitive Envionment[J]. Euorpean Joumal of Operational Research, 2005, 23(3): 121-129.

[2] Bates E. A Study of Passenger Transfer Facilities[J]. TRR, 1998: 662.

[3] Diekins, S.J.Ian. Park and Ride Facilities on Light Rail Transit Systems[J].Transportation, 2001: 23-36.

[4] Miclea. International tunnel fire-safety design practices[J]. ASHRAE Journal, 2007: 50-60.

[5] Shen Jingyan. Simplified Calculation for the Width of On and Off Region of Station Platform[J]. Urban Rapid Rail Transit, 2008, 21(5): 9-13.

[6] Tan Yu. Study on the theory of panning \& design for urban rail transit hub[D]. Doctoral Dissertation in Tongji University, 2002: 31-33.

[7] Wang Xiaorong, et al. On the Scale of Subway Stations[J]. Modern Tunnelling Technology, 2008, 45(3): 22-28.

[8] Wang Lihua. Preliminary Approach to Design of Subway Station Building[J]. Northern Communications, 2008, (2): 164-166.

[9] Yao Lan. Design for scale control of subway station[J]. Quality of Civil Engineering and Construction, 2006, (7): 48-51. 